American J. of Engineering and Applied Sciences 4 (1): 124-129, 2011

ISSN 1941-7020

(C) 2010 Science Publications

\title{
Bonding Property of Bituminous Crack Sealants in the Presence of Water
}

\author{
Ellie Fini and Taher Abu-Lebdeh \\ Department of Civil, Architectural and Environmental Engineering, \\ North Carolina A and T State University, NC 27411, Greensboro, USA
}

\begin{abstract}
Problem statement: Crack sealing and filling is the most widely used maintenance activity for in-service pavements. If an appropriate sealant material is selected and properly installed at the appropriate time of the pavement life, it retards pavement deterioration and increases its service life at a relatively low cost. However, in some cases it is reported that sealants failed prematurely, mainly due to environmental factors and water exposure. While there have been several studies on environmental factors, water related factors has not received appropriate attention. Nevertheless, various sealants have different water resistance property; hence, they perform differently when exposed to large amount of rain and humidity. Approach: Currently, there is no standard test method to evaluate sealant water resistance. Therefore, there is a need for a standard test method to precisely predict sealant performance when exposed to water. Such a test method can help examine various sealants in terms of their water resistance. This study introduces water conditioning procedure and a test method to measure bond strength of sealant under dry and wet condition. The effect of water on the Interfacial Fracture Energy (IFE) of bituminous sealants was measured by means of a blister test. This test allows the calculations of two fundamental parameters: tensile modulus and the Interfacial Fracture Energy (IFE). Results: Experimental results showed water exposure caused a significant drop in adhesion strength. However, no significant difference was observed between adhesion strength of specimens conditioned for 8 and $12 \mathrm{~h}$ of conditioning. Conclusion/Recommendations: The proposed approach and testing method can be used by the sealant manufacturers to improve their sealants' adhesion properties at the presence of water. However, further research works are needed to examine how sealant adhesion strength varies with water exposure duration. Also, the effect of water $\mathrm{pH}$ on sealant adhesion strength can be studied.
\end{abstract}

Key words: Sealant manufacturers, bond strength, interfacial fracture energy, blister test, water resistance, Strategic Highway Research Program (SHRP), Hot-Mix Asphalt (HMA), viscoelastic materials, axisymmetric debonding, First order Shear Deformable Theory (FSDT), pressurized blister, fundamental parameters

\section{INTRODUCTION}

Asphalt pavements, worldwide, continue to suffer serious distress problems (Masson et al., 2002; AlMutairi et al., 2009, Hasmadi and Taylor, 2008). In the USA, approximately $60 \%$ of the annual national transportation budget is spent on pavement maintenance. Crack sealing and filling is the most widely used maintenance activity for in-service pavements. This preventive maintenance activity is particularly favored among pavement agencies because it is inexpensive, quick and well-proven to delay the pavement deterioration caused by other mechanisms, such as weakening of subgrade and aggregate layers caused by water infiltration and stripping of Hot-Mix Asphalt (HMA) layers. If an appropriate sealant material is selected and properly installed at the appropriate time of the pavement life, it retards pavement deterioration and increases its service life at a relatively low cost. However, in some cases it is reported that sealants failed prematurely, mainly due to environmental factors and water exposure. As the result of newly concluded pooled-fund study on the Characterization of Bituminous Sealant, a PerformanceBased Guideline has been developed. In that effort which was sponsored by Federal Highway Administration and the US-Canadian Crack Sealant Consortium, many characteristics of bituminous

Corresponding Author:Taher Abu-Lebdeh,Department of Civil, Architectural and Environmental Engineering, North Carolina A and T State University, 1601 E. Market Street, Greensboro, NC 27411, Tel: (336) 334-7575 ext 664 Fax: (336) 334-7126 
sealants have been studied and several test methods and thresholds were developed (Al-Qadi et al., 2009; Masson et al., 2002).

While the effect of aging, temperature and curing on sealant performance has been considered in the aforementioned studies as well as in other related studies (Kumar and Vidivelli, 2010), the effect of water exposure on sealant performance has been neglected. It should be noticed that various sealants have different water resistance; hence they perform differently when exposed to large amount of rain and humidity. In a humid area with high percentage of annual precipitation rate, the problem is even more severe. The sealant failure during cold season is most noticeable because of cracks being wide open. In such cases the failure is mostly attributed to low temperature. However, the real failure may have been initiated much earlier during the warmer rainy season due to high amount of water exposure.

Adhesion is defined as the resistance against separation of two materials that are adhered together for a period of time at a specific temperature. The adhesion phenomenon has been studied from both physical and chemical points of view to improve adhesive bonds and predict failures. Adhesion is also an important parameter in many practical engineering applications, such as crack sealing. One well-known adhesive is bitumen, the basic material of the asphalt industry. Bitumen is the base of products such as polymermodified binde, hot-poured and cold-poured crack sealants and tack coats. Fracture is one of the main causes of adhesion failure. A fracture at the interface can be an irreversible entropy-creating process, through which a substantial amount of energy is dissipated. Energy dissipation is related to the ability of the interface to transfer stress and also to the adhesive's plastic and viscoelastic deformation properties. That is, crack will propagate if the energy available is equal to the energy required to extend the crack by a unit surface area. Since poor bonding results in cracks or delamination, fracture mechanics is a natural approach for characterizing the resistance to failure and for predicting the durability and performance of the bond.

Several researchers tried to quantify the adhesion through measuring the bond strength. Cheng et al. (2001) explained that each material has a unique energy attributed to its molecular interaction. They argued that the bond strength could be measured through the difference in surface energy between the components of the bond. They measured the surface energy of the asphalt and aggregates separately and calculated the adhesion strength of the bond through the Dupree equation. However, the adhesion strength in reality is generally several orders of magnitude higher than the adhesion strength derived through this approach. To develop a more realistic test, several mechanical tests were used to assess the adhesion of sealants to heat treated substrates (Masson et al., 2002). The principle of the tests was to confine the sealant between two aggregate pieces and apply tensile force to bring the assembly of sealant and aggregate to failure. The area under the load displacement curve was defined as the energy required to bring the assembly to failure and reported as adhesion strength. Masson and Zanzatto independently used this test to measure the adhesion strength of sealants to concrete (Muniandy et al., 2008). Masson measured energies of $200-500 \mathrm{~J} \mathrm{~m}^{-2}$ at $-37^{\circ} \mathrm{C}$, whereas Zanzatto obtained values of $500-1000 \mathrm{~J} \mathrm{~m}^{-2}$ at $-30^{\circ} \mathrm{C}$. At first look, these results show the bond strength was greater at $-30^{\circ} \mathrm{C}$ than at $-37^{\circ} \mathrm{C}$. However, due to its high dependence on geometry, this test should be restricted to studies of a single adhesive-substrate system using a single specimen size and geometry. Although this requirement can be met for examining the effect of curing time on the quality of the bond, it is more desirable to have a test that can measure some unique property of the bond that is independent of the geometry. Such a test equips the researcher with a unified sound method which can be repeated anywhere and produce comparable results.

In 1994, researchers in the Strategic Highway Research Program (SHRP) tried to develop a pressure loaded blister test. In this test, a thin layer of asphalt crack sealant was placed on top of an annular (donutshaped) disk of substrate (aggregate) and air was injected through the orifice in the substrate. They increased the pressure until the sealant was pushed away from the aggregate. They considered the peak pressure as a measure of adhesion and found that their test results based on this parameter did not correlate with field performance. In addition, they reported many of their tests failed cohesively. In other words, the sealant broke apart before separating from the substrate. Later, Fini and co-researchers reconsidered the test from another point of view. They found that cohesive failure could be prevented by using a thicker layer of adhesive and lowering the loading rate. In addition, they used alcohol instead of pressurized air which was used earlier in SHRP study. They showed if the right test parameter is selected, the test results can be independent of the geometry, therefore, a thicker layer of adhesive will not affect the test result. However, in order to measure adhesion correctly, the blister profile's evolvement needs to be recorded along with the blister pressure. They showed peak pressure alone is not independent of geometry and cannot serve as a measure 
of adhesion (Fini et al., 2009). They measured Interfacial Fracture Energy (IFE) which is a fundamental property of the interface as a measure of sealant-aluminum adhesion strength.

\section{MATERIALS AND METHODS}

In the present study, the proposed test program implements the principles of fracture mechanics in a pressurized circular blister test. The apparatus is specifically designed to conduct the test for bituminous crack sealant or other bitumen-based materials. In this test, a fluid is injected at a constant rate at the interface between the substrate and the adhesive to create a blister. This test provides a means to quantify important factors, such as the moisture susceptibility of a bond and can determine the elastic modulus of the sealant and its residual stresses.

Most current sealant test methods are empirical and only provide a qualitative measure of bond strength, in addition to their limitations in predicting accurately the sealant adhesive failure. In this study, blister test method is used to measure Interfacial Fracture Energy (IFE) of sealant at dry and wet condition. This test is selected because it measures a fundamental property of the interface while counting for bulk properties of sealants. Furthermore, due to its substrate annular shape, water conditioning can be conducted without submerging the sealant itself. In other word, water can reach the interface without soaking the sealant. Therefore, effect of water on the interface can be examined properly. In traditional water conditioning methods, specimen is submerged in water. However, submerging the specimen can cause sealant to swell and change sealant's bulk properties as well as those of interface. Therefore, bond strength measured after this type of conditioning can be misleading.

In the presented conditioning procedure, only the interface is exposed to water through the orifice and the notch which is created at the interface. To do so, blister test specimens (Fig. 1) were prepared following the procedure developed by the author and co-investigators in 2008. Each specimen is composed of an annular (donut-shaped) aluminum substrate plate covered with sealant on one side. The substrate was an annular disk with inner and outer diameters of 25 and $125 \mathrm{~mm}$, respectively. The inner whole (orifice) in the substrate is sealed with a close-fitted aluminum plug flush with the substrate surface. It should be mentioned that, prior to samples' preparation, the aluminum substrate is washed with water and cleaning solvent and then airdried. Several cleaning approaches were tried and the utilized one was found acceptable.

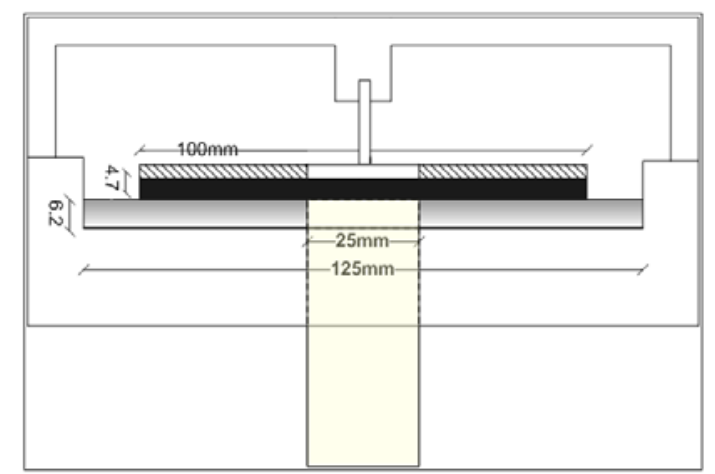

Fig. 1: Blister test apparatus and inflated sealant

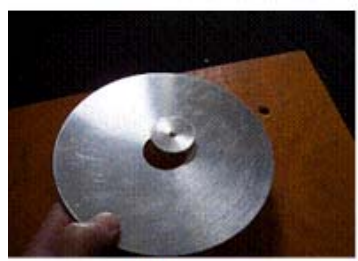

(a)

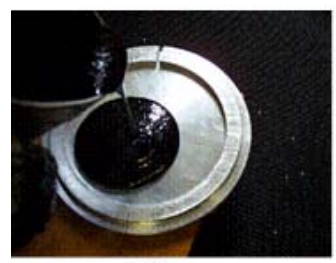

(c)

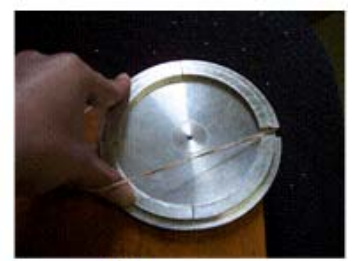

(b)

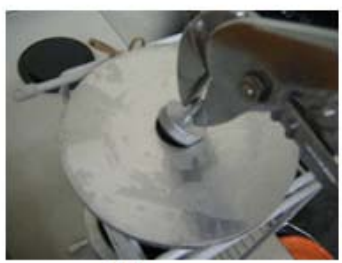

(d)
Fig. 2: (a), assembling the Plug and the FEP; (b) Assembling the Molds and Holding them with a Rubber Band; (c) Pouring Sealant onto the Aluminum Substrate; (d) Removing the Plug and creating the notch

To prepare specimen a 4-pieces mold (Fig. 2) was assembled directly on top of the substrate and filled with sealant. To facilitate water conditioning a notch was created at the interface between sealant and aluminum plate. To create the notch, a 0.09-mm-thick and 27-mm-diameter disc shape transparent Fluoropolymer (FEP) film was placed on top of the plug before casting the sealant. After placing the film, a silicon-based release agent is sprayed on top of the film so that the film does not stick to the binder or sealant cast on the plug. Because the film has an adhesive backing, it sticks to the plug. The film and plug are removed from the specimen prior to testing. This will create a small notch at the interface which allows water to reach the interface directly (Fig. 2). 
Specimens were trimmed after $60 \mathrm{~min}$ and placed in cooling bath (at $\left.-22^{\circ} \mathrm{C}\right)$ for $45 \mathrm{~min}$. Then the aluminum plugs are removed and specimens are ready for conditioning and testing. Three sets of specimen were prepared each in two replicates. One set was tested without conditioning. The other two sets were placed in water bath at room temperature for 8 and $12 \mathrm{~h}$ respectively. Water depth was adjusted so that it does not cover the specimen and the only exposure of interface to sealant occurs through the orifice and the notch.

To conduct the test, a servo-hydraulic pump was used to displace a piston at a constant rate. The upward movement of the piston injects alcohol at a constant rate of $0.1 \mathrm{~L} \mathrm{~h}^{-1}$ through a channel that is connected to the specimen (Fig. 1). Continuous injection of alcohol pushes sealant away from the substrate creating a blister which continues to grow until the adhesive separates from the substrate. The induced pressure inside the blister was measured with a Viatran pressure transducer. The transducer resolution is 0.25 percent of full range and its maximum capacity is $3.5 \mathrm{MPa}$ (500psi). The height of the evolving blister is recorded through a linear variable displacement transducer of \pm $12 \mathrm{~mm}$. The fluid pressure and blister height are measured as functions of time and used to calculate Interfacial Fracture Energy (IFE), which is a fundamental property that can be used to predict adhesion. That is, the pressure continued increasing until it reached its maximum (peak) level at which sealant started debonding from the aluminum plate and pressure dropped. The peak pressure was recorded and reported as the interfacial adhesion between the sealant and the aluminum. It should be noted that although the fluid injection rate is constant, the debonding rate may not be constant because of the specimen's geometry and the axisymmetric debonding process.

\section{RESULTS}

The blister test results are recorded in terms of blister height and pressure as shown in Fig. 3. The prepeak region shows linear behavior followed by gradual decrease rather than sharp drop indicating strong bond between the sealant and aluminum substrate plate. The behavior in the pre-peak region is related to the adhesive behavior before any debonding begins. This part of the curve can be used to study the properties of the adhesive layer independently of the descending post-peak curve, which is related to the interface. Further, it was noticed that the blister forms and its radius remain fixed until a critical "peak" pressure is reached. At this peak pressure value, the radius of the blister increases in size, indicating the start of the debonding process at the interface. Further, the linear function of pressure vs. blister height in the pre-peak region suggests that the blister behaves like a plate. This is because of the fact that if pressure is a cubic function of the blister height, the blister's behavior can be analyzed as a membrane. However, if there is a linear relationship between pressure and blister height, which is the case in this study, the blister exhibits plate behavior.

Assuming plate behavior for sealant and incorporating plate theory through energy balance analysis, the sealant modulus (E) may be determined from the measured values of the fluid pressure as presented in Equation 1. The Interfacial Fracture Energy (IFE), which is an indication of bond strength, can be extracted from the test data using Mindlin First order Shear Deformable Theory (FSDT) (Wang et al., 2000; Ali et al., 2010; Zlender, 2008 ), as shown in Eq. 2:

$$
\begin{aligned}
& E(t)=\frac{p(t)}{d(t)}\left[\frac{\left(1-v^{2}\right) a^{4}}{5.3 h^{3}}+\frac{(1+v) a^{2}}{1.7 h}\right] \\
& I F E=\frac{p^{2}(t) a}{E(t)}\left[\frac{3 a^{3}\left(1-v^{2}\right)}{32 h^{3}}+\frac{3 a(1+v)}{10 h}+\frac{2\left(1-v^{2}\right)}{\pi}\right]
\end{aligned}
$$

Where:

$\mathrm{d}(\mathrm{t})=$ The adhesive deflection at a distance

$\mathrm{t}=$ From the center of the circular film

$\mathrm{p}=$ The pressure inside the blister

$\mathrm{a}=$ The radius of the orifice

$\mathrm{v}=$ The Poisson ratio

$\mathrm{E}=$ the sealant modulus and $\mathrm{h}$ is the film thickness

The first term of this equation accounts for the bending while the second term counts for shear displacement of the sealant film under axis-symmetric uniform pressure.

Following this approach, the adhesion strength of the selected sealant to aluminum at $-22^{\circ} \mathrm{C}$ (Fig. 4) after and before water conditioning was calculated. To do so, peak pressure and corresponding height of the blister were inserted into Equation 2. Poisson ratio was assumed to be 0.35 and sealant modulus was calculated separately from data recorded before the peak using Eq. 1. The IFE parameter may be defined as the energy required to separate a unit area of the interface. As would be expected, the IFE was nearly the same during the debonding period because it is independent of geometry. However, it is also important to note that it may depend on surface preparation. Therefore, the surface preparation needs to remain constant if IFE is to be used to predict adhesion for various geometries. 


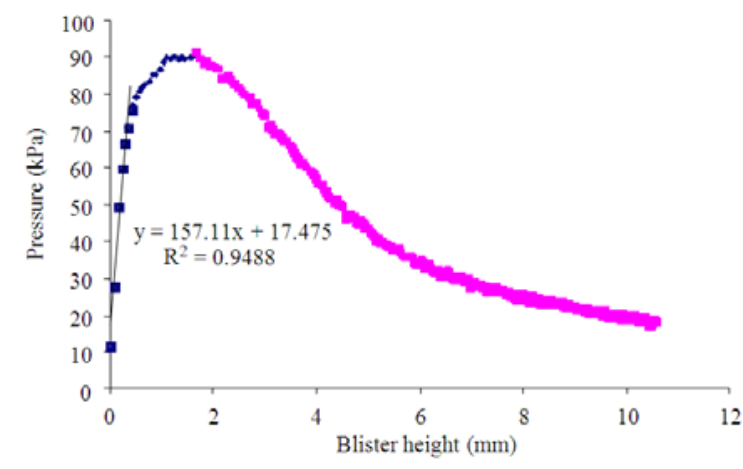

Fig. 3: Pressure Vs. Blister Height, Before and During Debonding

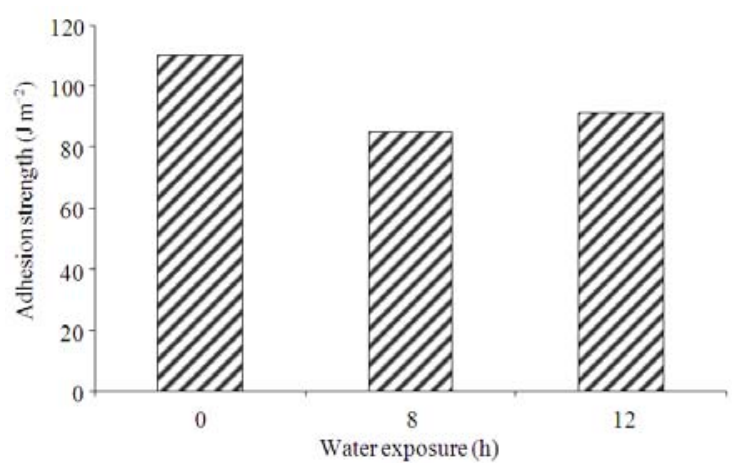

Fig. 4: Adhesion decreases due to water exposure

Effect of Water Exposure on Adhesion: The blister test was used to examine the effect of water exposure (conditioning) on adhesion strength and consequently on quality of the bond. To conduct the tests, three sets of specimens were prepared. One set was used as control specimen (no conditioning) the other two sets were placed in a water bath at room temperature for 8 and 12 h. The water depth was adjusted so that it does not cover the sealant. Therefore, the only exposure to water occurs at the interface and through the notch. Experimental results showed water exposure caused a significant drop in adhesion strength. However, no significant difference was observed between adhesion strength of specimens conditioned for 8 and 12 h as shown in Fig. 4).

\section{DISCUSSION}

Currently, there is no standard test method to evaluate sealant's water resistance. Therefore, there is a need for a standard test method and specification which is based on fundamental sealant property and can precisely predict sealant performance when exposed to water. Such a test method can assist researchers and practitioners to examine various sealants in terms of their water resistance. That will allow practitioners and maintenance crew to select right sealant based on the annual precipitation rate and humidity level in their area and to ensure sealant performs well in such environment. This study, introduces a procedure for water conditioning and a test method to measure bond strength of sealant under dry and wet conditions. The effect of water on the Interfacial Fracture Energy (IFE) of bituminous sealants was measured by means of a blister test. The substrate was a smooth aluminum, replacing aggregate substrate because it has similar thermal coefficient to that of aggregates, in addition to its compatibility with sealant, controllable roughness, high resistance to extreme temperatures and availability.

The blister test allows calculating two fundamental parameters: tensile modulus and the Interfacial Fracture Energy (IFE). Since week bonding results in cracks or delamination; hence, fracture mechanics is a natural approach for characterizing the resistance to failure and for predicting the durability and performance of the bond. The Interfacial Fracture Energy (IFE) can be extracted easily through this test. Sealant modulus (E (t)) is another parameter that can be determined from the blister test. Knowing the relation between pressure and blister height (Fig. 3), the modulus, E (t) can be calculated during bulging (pre-peak pressure). The modulus can only be defined up to the peak pressure because after peak, the boundary condition starts changing as debonding continues. However, for more precise measurement of the modulus, debonding should not be allowed during the bulging period. This can be achieved by confining the adhesive film. Further, since sealants are viscoelastic materials, their time dependent modulus can be calculated as a function of time and allows the calculation of IFE at the start of debonding. In this study, the modulus calculated at the peak pressure was used to calculate the IFE using Equation 3. Determination of IFE was based on the balance between the potential energy provided by the externally applied pressure and the elastic strain energy stored in the adhesive. It should be noted that, since IFE is a geometry independent value, it remains nearly the same as the debonding continues.

Examining Fig. 3, one can observe that the prepeak curve fits well to a linear function $\left(\mathrm{R}^{2}=95 \%\right)$. This implies that in this range of deformation, plate behavior is dominant. The deviation of the data from the straight line is due to both softening and viscoelastic behavior of the sealant near the peak pressure. This may be due to stress relaxation which occurs during bulging (prepeak), the slope of the pressure-blister height curve, which is related to the sealant's modulus, also decreases (Al-Qadi et al., 2009). Using the relationship between 
bending solution of Classical Plate Theory (CPT) and Mindlin First order Shear Deformable Theory (FSDT), equations 2 and 3 account for the shear effect. Having IFE as a system parameter and Young's modulus (E) of the adhesive material, one can predict adhesive failure in complex adhesive joints by stress analysis and energy balance of the geometry (Al-Qadi et al., 2009).

\section{CONCLUSION}

This study uses a pressurized blister test to measure adhesion strength of sealant to aluminum under wet and dry conditions. Quantifying the adhesion strength under wet and dry condition, this study found water as an effective factor on sealant adhesion strength and consequently sealant performance. It was shown that water exposure can reduce sealant adhesion strength significantly. However, no significant difference was found between 8 and $12 \mathrm{~h}$ of conditioning. The proposed approach can be helpful when selecting crack sealants for various climatic and geologic regions. In addition, sealant manufacturers can use the test to improve their sealants' adhesion properties at the presence of water. Further research needs to be conducted to examine how sealant adhesion strength varies with water exposure duration. In addition, effect of water $\mathrm{pH}$ on sealant adhesion strength can be studied.

\section{ACKNOWLEDGEMENT}

The researchers would like to acknowledge the assistance and support provided by Dr. Imad Al-Qadi from University of Illinois who provided guidance as well as access to Advanced Transportation Research and Engineering Laboratory. The author also would like to thank the invaluable consultation and support of Dr. Lynn Penn from Drexel University and Dr. Ernie Bastian from Federal Highway Administration.

\section{REFERENCES}

Ali, A., M. Hosseini and B.B. Sahari, 2010. A review of constitutive models for rubber-like materials. Am. J. Eng. Applied Sci., 3: 232-239. DOI: 10.3844/ajeassp.2010.232.239
Al-Mutairi, N., F. Al-Rukaibi and P. Koushki, 2009. Measurements and model calibration of urban traffic noise pollution. Am. J. Environ. Sci., 5: 613-617. DOI: 10.3844/ajessp.2009.613.617

Al-Qadi,I.L., S.H. Yang, E. Fini, J.F. Masson and K. McGhee, 2009. Performance based specification guidelines for the selection of bituminous-based hot-poured crack sealants. J. Assoc. Asphalt Paving Technologists, 78: 491-532.

Cheng, D.X., D. Little, R.L. Lytton and J.C. Holste, 2001. Surface free energy measurement of aggregates and its application to adhesion and moisture damage of asphalt-aggregate systems. Proceeding of the International Center for Aggregates Research, 9th Annual Symposium, Austin, TX, 2001. http://www.ecst.csuchico.edu/_depts/ce/Faculty_Staff/ Members/Ding_Cheng.html

Fini, E., I.L. Al-Qadi, J.F. Masson and K.K. McGhee, 2009. Interfacial fracture energy: an indicator of bituminous materials adhesion. J. Assoc. Asphalt Paving Technol., 77: 827-850.

Kumar, D.R. and B. Vidivelli, 2010. Acrylic rubber latex in ferrocement for strengthening reinforced concrete beams. Am. J. Eng. Applied Sci., 3: 277-285. DOI: 10.3844/ajeassp.2010.277.285

Masson, J.F., P. Collins, J.F. Margeson and G. Polomark, 2002. Analysis of bituminous crack sealants by physicochemical methods: Relationship to field performance. J. Trans. Res. Board 1795: 33-39. DOI: $10.3141 / 1795-04$

Muniandy, R., H. Jafariahangari, R. Yunus and S. Hassim, 2008. Determination of rheological properties of bio mastic asphalt. Am. J. Eng. Applied Sci., 1: 204-209. DOI: 10.3844/ajeassp.2008.204.209

Wang, C.M., J.N. Reddy and K.H. Lee, 2000. Shear Deformable Beams and Plates. 1st Edn., Elsevier, Amsterdam, ISBN-13: 9780080437842, pp: 296.

Zlender, B., 2008. Prediction of permanent deformation of pavement's unbounded layers based on cyclic triaxial tests. Am. J. Applied Sci., 5: 22-28. DOI: 10.3844/ajassp.2008.22.28 